\title{
EFEITOS DO THIDIAZURON E DO ÁCIDO GIBERÉLICO NAS CARACTERÍSTICAS DOS CACHOS DE UVA DE MESA CULTIVAR RUBI, NA REGIÃO DA NOVA ALTA PAULISTA ${ }^{1}$
}

\author{
RENATO VASCONCELOS BOTELHO ${ }^{2}$, ERASMO JOSÉ PAIOLI PIRES ${ }^{3}$, \\ MAURILO MONTEIRO TERRA ${ }^{3}$, STELLA CONSORTE CATO ${ }^{4}$
}

\begin{abstract}
RESUMO- Com o objetivo de avaliar os efeitos de thidiazuron e de ácido giberélico nas características dos cachos de uvas 'Rubi', foi conduzido um experimento, utilizando-se de thidiazuron a 5 e $10 \mathrm{mg} . \mathrm{L}^{-1}$ e ácido giberélico a 20mg.L ${ }^{-1}$, combinados ou não. As aplicações dos produtos foram realizadas aos 14; 21 ou 28 dias após o pleno florescimento, por meio de imersão dos cachos. Todos os tratamentos com reguladores de crescimento aumentaram a massa dos cachos. A massa dos bagos e dos engaços foi identicamente influenciada pela aplicação dos produtos, porém menos evidente, quando as aplicações foram realizadas aos 28 dias após o pleno florescimento. As aplicações de thidiazuron a $5 \mathrm{mg} . \mathrm{L}^{-1}$, aos 14 ou 21 dias após o florescimento, não diferiram das aplicações de ácido giberélico para as variáveis estudadas. Não houve diferenças significativas para as variáveis teor de sólidos solúveis totais, acidez titulável, porém os tratamentos com thidiazuron retardaram a maturação em até 7 dias.
\end{abstract}

Termos para indexação: videira, Vitis vinifera, citocinina, giberelina, regulador de crescimento.

\section{EFFECTS OF THIDIAZURON AND GIBBERELLIC ACID ON THE CLUSTER CHARACTERISTICS OF RUBI TABLE GRAPE CULTIVAR, ON THE REGION OF NOVA ALTA PAULISTA.}

\begin{abstract}
With the objective of evaluating the effects of thidiazuron and gibberellic acid on the cluster characteristics of 'Rubi' grapes, a trial was conducted where was used thidiazuron at 5 and $10 \mathrm{mg} . \mathrm{L}^{-1}$ and gibberellic acid at $20 \mathrm{mg} . \mathrm{L}^{-1}$, associated or not . The products were applied in three epochs: 14, 21 or 28 days after full bloom, by dipping the clusters. All treatments with growth regulators increased the cluster weight. The berries and rachis weight were identically influenced, but it was less evident when the applications were done 28 days after full bloom. Applications of thidiazuron at $5 \mathrm{mg} . \mathrm{L}^{-1}, 14$ or 21 days after full bloom, didn't differ to the gibberellic acid applications for the variables in studying. There weren't differences in the variables contents of soluble solutes, titrable acidity, although the treatments with thidiazuron delayed cluster ripening until 7 days.
\end{abstract}

Index terms: vine, Vitis vinifera, , cytokinin, gibberellin, growth regulator.

O thidiazuron (TDZ: N-fenil-N-1,2,3-tidiazol-5-tiuréia) é uma citocinina sintética que, no Brasil, é largamente utilizada na cultura do algodoeiro para provocar desfolhamento. Em fruticultura, há trabalhos que comprovaram a eficiência do thidiazuron na quebra de dormência da macieira e pereira (Araújo et al., 1991; Francisconi et al., 1982), e no aumento do tamanho e pegamento dos frutos de maçãs, kiwis e uvas (Petri et al., 1992; Reynolds et al., 1992).

$\mathrm{O}$ ácido giberélico $\left(\mathrm{AG}_{3}\right)$ é o regulador de crescimento mais amplamente utilizado na viticultura em nível comercial, visando principalmente ao aumento do tamanho dos bagos. Entretanto, para Retamales et al. (1995), a busca de uma alternativa para o uso de ácido giberélico é importante, tendo em vista que este apresenta alguns efeitos indesejáveis como: redução da fertilidade de gemas, aumento do vigor das plantas, degrana dos cachos pós-colheita e maior suscetibilidade dos frutos às podridões.

Tendo em vista as poucas pesquisas desenvolvidas com thidiazuron em videiras, este trabalho teve como objetivo verificar os efeitos de thidiazuron e ácido giberélico nas características dos cachos de uvas de mesa 'Rubi'.

$\mathrm{O}$ experimento foi realizado em vinhedo comercial da cultivar Rubi, localizado no município de Ouro Verde, região Oeste do Estado de São Paulo. As videiras, enxertadas sobre portaenxerto IAC-572 Jales, com 6 anos de idade, estavam conduzidas no sistema de latada, no espaçamento de $6 \times 3 \mathrm{~m}$.

Os tratos culturais, exceto a utilização de ácido giberélico, foram idênticos ao sistema convencional adotado pelo produtor. As aplicações de reguladores de crescimento foram efetuadas através de única imersão dos cachos em solução aquosa, adicionada de espalhante adesivo Iharagen-Sâ a 1\%, contida em recipiente plástico.

1 (Trabalho 068/2001). Recebido: 19/03/2001. Aceito para publicação: 05/10/2001.

2 Eng $^{\circ} \mathrm{Agr}^{\circ}$ Doutorando do curso de pós-graduação FCA-UNESP, Campus de Botucatu-SP, bolsista da FAPESP. IAC-Centro de Fruticultura, C. Postal 28, CEP 13001-970, Campinas-SP. E-mail:rebotelho@hotmail.com.

$3 \mathrm{Eng}^{\mathrm{o}} \mathrm{Agr}^{\circ} \mathrm{Dr}$., Pesquisador Científico. IAC-Centro de Fruticultura, Bolsista do CNPq

4 Eng $^{\text {a }}$ Agr $^{\mathrm{a}}$ Mestranda do curso de pós-graduação, ESALQ-USP, bolsisita FAPESP. 
Para os tratamentos, foi utilizado thidiazuron a 5 ou 10 mg. $\mathrm{L}^{-1}$, e ácido giberélico a $20 \mathrm{mg} . \mathrm{L}^{-1}$, associados ou não. As aplicações foram realizadas em três épocas diferentes: aos 14; 21 ou 28 dias após o pleno florescimento.

O delineamento experimental foi em blocos ao acaso, com 16 tratamentos, 6 repetições e parcela constituída de dois cachos. Plantas de videiras foram selecionadas para aplicação dos tratamentos em cada linha do bloco, de forma que tivessem o mesmo vigor.

A coleta dos cachos foi realizada no ponto de colheita comercial, isto é, quando atingiram teor de sólidos solúveis totais mínimo de $14,0^{\circ}$ Brix, sendo iniciada em 4 de setembro de 2000 , portanto aos 157 dias após a poda realizada em 27 de março de 2000. Posteriormente, os cachos foram acondicionados em sacos de polietileno, devidamente identificados e levados para laboratório para a realização das avaliações.

Foram avaliadas para cada cacho as seguintes variáveis: massa do cacho, bagos e engaço, em balança de precisão; comprimento e largura do cacho e bagos (subamostra de 20 bagos), com paquímetro; número de sementes por bago (subamostra de 20 bagos); ciclo entre poda e colheita,em número de dias; teor de sólidos solúveis totais, por refratometria; e acidez total titulável, por tilulação com $\mathrm{NaOH} 0,1 \mathrm{~N}$ (Carvalho et al., 1990).

Os dados do experimento foram submetidos à análise de variância e comparação entre médias, pelo teste de Duncan, a $5 \%$ de probabilidade.

Todos os tratamentos com reguladores de crescimento aumentaram significativamente a massa dos cachos. Este efeito foi menos pronunciado quando as aplicações foram realizadas aos 28 dias após o florescimento (Tabela 1).

Para a variável comprimento dos cachos, não houve diferenças significativas, enquanto a largura dos cachos foi significativamente aumentada para alguns tratamentos. Entretanto, constatou-se, para estas variáveis, maiores valores para quase todos os tratamentos com reguladores de crescimento (Tabela 1).
A massa dos bagos foi incrementada para todos os tratamentos com reguladores de crescimento, excetuando-se a aplicação de thidiazuron a $5 \mathrm{mg} . \mathrm{L}^{-1}, 28$ dias após pleno florescimento. Quando os reguladores de crescimento foram utilizados de forma isolada, os melhores resultados para esta variável foram verificados para aplicações aos 14 e 21 dias. Segundo Ben Tal (1990), aplicações muito tardias de $\mathrm{AG}_{3}$, em uvas da cultivar Thompson Seedless, contribuíram muito pouco para o aumento do tamanho dos bagos.

As aplicações de thidiazuron a $5 \mathrm{mg} \cdot \mathrm{L}^{-1}$, aos 14 ou 21 dias após pleno florescimento, não diferiram significativamente, para as variáveis massa de cachos e bagos, das aplicações com ácido giberélico ou thidiazuron a $10 \mathrm{mg} . \mathrm{L}^{-1}$, assim como não houve efeito sinérgico dos dois reguladores de crescimento, em aplicações combinadas.

Para os resultados de comprimento e largura dos bagos, poucos tratamentos com reguladores de crescimento diferiram da testemunha, porém a maioria deles apresentou valores superiores (Tabela 2).

Reynolds et al. (1992) também verificaram aumento da massa de cachos e bagos, em quatro variedades de uvas sem sementes, através de aplicações de citocininas sintéticas do grupo das feniluréias, como o thidiazuron e o CPPU. Miele et al. (2000) obtiveram resultados semelhantes em uvas 'Itália' pela aplicação de CPPU a 5mg. $\mathrm{L}^{-1}$ ou $\mathrm{AG}_{3}$ a $40 \mathrm{mg} \cdot \mathrm{L}^{-1}$.

A maioria dos tratamentos apresentou tendência de redução do número de sementes por bago, sendo que a aplicação de thidiazuron a $5 \mathrm{mg} . \mathrm{L}^{-1}$ associado a $\mathrm{AG}_{3}$, aos 14 dias após pleno florescimento, diferiu significativamente da testemunha. Estes resultados estão de acordo com aqueles obtidos por Petri et al. (1992), em maçãs tratadas com thidiazuron, estando este efeito relacionado ao desenvolvimento de frutos partenocárpicos.

A massa dos engaços foi significativamente aumentada pela aplicação de reguladores de crescimento, excetuando-se os tratamentos realizados aos 28 dias após pleno florescimento (Tabela 1).

TABELA 1 - Massa, comprimento e largura de cachos; massa dos engaços e número de sementes por bago de uvas da cultivar Rubi tratadas com thidiazuron e ácido giberélico, 2000.

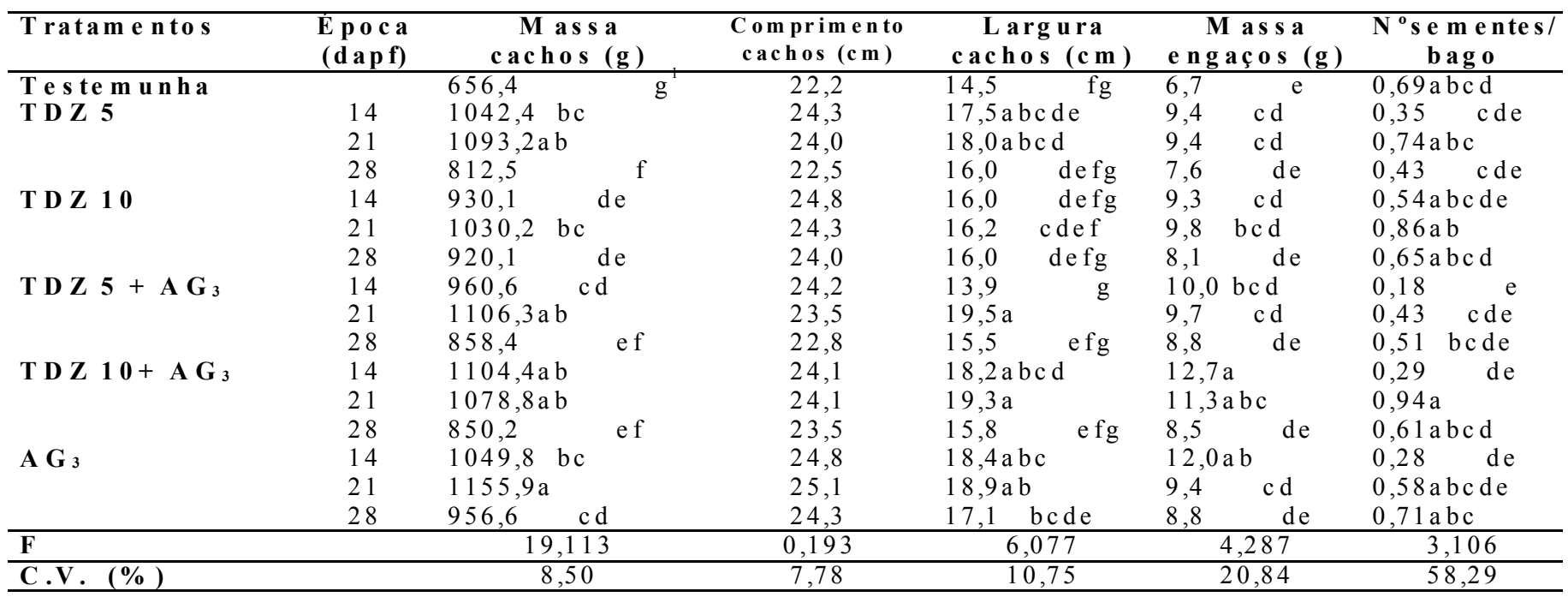

${ }^{1}$ Médias seguidas pela mesma letra, numa mesma coluna, não diferem entre si, pelo teste de Duncan, ao nível de $5 \%$ de probabilidade.

dapf $=$ dias após pleno florescimento, TDZ $5=5 \mathrm{mg} \cdot \mathrm{L}^{-1}$, TDZ $10=10 \mathrm{mg} \cdot \mathrm{L}^{-1} \mathrm{AG}_{3}=20 \mathrm{mg} \cdot \mathrm{L}^{-1}$ 
TABELA 2 - Massa, comprimento e largura dos bagos; sólidos solúveis totais, acidez total titulável e ciclo de uvas da cultivar Rubi tratadas com thidiazuron e ácido giberélico, 2000.

\begin{tabular}{|c|c|c|c|c|c|c|c|}
\hline Tratamentos & $\begin{array}{r}\text { E poca } \\
(\text { d a p f })\end{array}$ & $\begin{array}{c}\text { M ass a } \\
\text { bagos }(\mathrm{g})\end{array}$ & $\begin{array}{c}\text { Comprimento } \\
\text { bagos }(\mathrm{m} \mathrm{m})\end{array}$ & $\begin{array}{c}\text { Largura } \\
\text { bagos }(\mathrm{m} \mathrm{m})\end{array}$ & SST (\%) & $\begin{array}{c}\text { A T T } \\
(\mathrm{g} / 100 \mathrm{~m} \mathrm{l})\end{array}$ & $\begin{array}{l}\text { Ciclo } \\
\text { (dias) }\end{array}$ \\
\hline Testemunha & & $6,21 \quad f$ & $26,2 \quad \mathrm{c}$ & $22,3 \quad b c$ & $15,5 \mathrm{abc}$ & 0,601 & $141,4 \quad \mathrm{~cd}$ \\
\hline \multirow[t]{3}{*}{ T D Z 5} & 14 & $8,74 \mathrm{abc}$ & $29,4 \mathrm{ab}$ & $23,5 \mathrm{abc}$ & $15,0 \quad \mathrm{bc}$ & 0,592 & $147,2 \mathrm{ab}$ \\
\hline & 21 & $8,24 \mathrm{abcde}$ & $28,1 \mathrm{abc}$ & $23,5 \mathrm{abc}$ & $15,7 \mathrm{ab}$ & 0,631 & $145,7 \mathrm{abc}$ \\
\hline & 28 & 7,15 e f & $26,6 \quad c$ & $21,9 \quad \mathrm{c}$ & 14,7 bc & 0,589 & $148,6 \mathrm{a}$ \\
\hline \multirow[t]{3}{*}{ T D Z 10} & 14 & $8,66 \mathrm{abcd}$ & $28,7 \mathrm{abc}$ & $23,8 \mathrm{ab}$ & $14,7 \quad \mathrm{bc}$ & 0,632 & $147,1 \mathrm{ab}$ \\
\hline & 21 & $8,87 \mathrm{ab}$ & $29,3 \mathrm{ab}$ & $23,6 \mathrm{ab}$ & $15,7 \mathrm{ab}$ & 0,593 & $145,7 \mathrm{abc}$ \\
\hline & 28 & 8,10 bc de & $29,3 \mathrm{ab}$ & $22,6 \mathrm{bc}$ & $15,5 \mathrm{ab}$ & 0,595 & $148,5 \mathrm{a}$ \\
\hline \multirow[t]{3}{*}{$\mathbf{T} \mathbf{D} \mathbf{Z}+\mathbf{A ~ G}_{3}$} & 14 & 8,00 bcde & $28,0 \mathrm{abc}$ & $23,5 \mathrm{abc}$ & $14,7 \quad \mathrm{bc}$ & 0,609 & $145,7 \mathrm{abc}$ \\
\hline & 21 & $8,36 \mathrm{abcde}$ & $27,7 \mathrm{abc}$ & $23,3 \mathrm{abc}$ & $13,8 \quad \mathrm{c}$ & 0,631 & $144,3 \mathrm{abcd}$ \\
\hline & 28 & $8,15 \mathrm{abcde}$ & $28,3 \mathrm{abc}$ & $23,7 \mathrm{ab}$ & 14,5 bc & 0,664 & $145,7 \mathrm{abc}$ \\
\hline \multirow[t]{3}{*}{$\mathrm{TDZ}_{\mathbf{1 0}}+\mathrm{AG}_{3}$} & 14 & $7,38 \quad \mathrm{de}$ & $27,9 \mathrm{abc}$ & $22,8 \quad \mathrm{bc}$ & 14,3 bc & 0,593 & $147,1 \mathrm{ab}$ \\
\hline & 21 & $9,41 \mathrm{a}$ & $30,0 \mathrm{a}$ & $24,6 \mathrm{a}$ & $16,8 \mathrm{a}$ & 0,613 & $144,3 \mathrm{abcd}$ \\
\hline & 28 & $8,21 \mathrm{abcde}$ & $28,0 \mathrm{abc}$ & $23,5 \mathrm{abc}$ & $15,0 \quad \mathrm{bc}$ & 0,596 & $145,7 \mathrm{abc}$ \\
\hline \multirow[t]{3}{*}{$\mathbf{A} \mathbf{G}_{3}$} & 14 & $8,08 \mathrm{bcde}$ & $28,2 \mathrm{abc}$ & $22,5 \mathrm{bc}$ & $15,4 \mathrm{abc}$ & 0,621 & 142,8 bcd \\
\hline & 21 & $8,01 \mathrm{bcde}$ & $27,9 \mathrm{abc}$ & $23,1 \mathrm{abc}$ & $14,5 \mathrm{bc}$ & 0,652 & 142,8 \\
\hline & 28 & $7,43 \quad \mathrm{de}$ & $27,9 \mathrm{abc}$ & $23,4 a b c$ & $15,1 \mathrm{abc}$ & 0,671 & 140,0 \\
\hline $\mathbf{F}$ & & 3,141 & 1,670 & 1,809 & 1,506 & 1,076 & 2,2901 \\
\hline C.V. (\%) & & 12,75 & 5,84 & 4,72 & 9,13 & 13,08 & 2,94 \\
\hline
\end{tabular}

${ }^{1}$ Médias seguidas pela mesma letra, numa mesma coluna, não diferem entre si, pelo teste de Duncan, ao nível de $5 \%$ de probabilidade. dapf $=$ dias após pleno florescimento, TDZ $5=5 \mathrm{mg} \cdot \mathrm{L}^{-1}, \mathrm{TDZ} 10=10 \mathrm{mg} \cdot \mathrm{L}^{-1} \mathrm{AG}_{3}=20 \mathrm{mg} \cdot \mathrm{L}^{-1}$

Não ocorreram diferenças significativas para as variáveis teor de sólidos solúveis totais e acidez total titulável, porém houve aumento do ciclo entre poda e colheita para a maioria dos tratamentos com thidiazuron, sendo que esta extensão foi no máximo de 7 dias, em média, em relação à testemunha (Tabela 2). Leão et al. (1999) também constataram atraso de 8 dias na maturação de uvas 'Perlette' pela aplicação de CPPU associado a $\mathrm{AG}_{3}$.

Pelos resultados aqui apresentados, ficou evidente a possibilidade de utilização de thidiazuron visando ao aumento do tamanho dos bagos de uvas 'Rubi', não diferindo significativamente da aplicação convencional com $\mathrm{AG}_{3}$. A dose de $5 \mathrm{mg} . \mathrm{L}^{-1}$ de thidiazuron, aplicado entre 14 e 21 dias após pleno florescimento, foi suficiente para obtenção de bagos e cachos de tamanhos adequados para comercialização. $\mathrm{O}$ efeito deste regulador de crescimento no atraso da maturação dos frutos foi de, no máximo, 7 dias, podendo ser uma estratégia interessante para o viticultor, visando principalmente ao maior escalonamento da colheita.

\section{REFERÊNCIAS BIBLIOGRÁFICAS}

ARAÚJO, M.M.; FORTES, G.R.; SANTOS FILHO, B.G. Thidiazuron - uma alternativa para superar a dormência de gemas de macieira (Malus domestica Borkh). Revista Brasileira de Fruticultura, Cruz das Almas, v.13, n.3, p.249-253, 1991.

BEN TAL, Y. Effects of gibberellin treatments on ripening and berry drop from Thompson Sedless grapes. American Journal of Enology and Viticulture, Davis, v.41, n.2, p.142-146, 1990.

CARVALHO, C.R.L.; CARVALHO, P.R.N.; MANTOVANI, D.M.B.;
MORAES, R.M. Análise química de alimentos. Campinas: ITAL, 1990. $121 \mathrm{p}$.

FRANCISCONI, A.H.D.; BARRADAS, C.I.N.; MARODIN, G.A.B.; SEIBERT, E. Efeito de óleo mineral, cianamida hidrogenada e thidiazuron na quebra de dormência e produção da pereira (Pyrus communis L.) cv. Packam's Triumph. Revista Brasileira de Fruticultura, Cruz das Almas, v.14, n.1, p.161-166, 1992.

LEÃO, P.C.S.; LINO JUNIOR, E.C.; SANTOS, E.S. Efeitos do CPPU e ácido giberélico sobre o tamanho de bagas da uva Perlette cultivada no vale do São Francisco. Revista Brasileira de Fruticultura, Jaboticabal, v.21, n.1, p.74-78, 1999.

MIELE, A.; RIZZON, L.A.; DALL'AGNOL, I. Efeito de reguladores de crescimento no tamanho da baga e na composição do mosto da uva 'Itália'. Revista Brasileira de Fruticultura, Jaboticabal, v.22, n.2, p.272-276, 2000.

PETRI, J.L.; ARGENTA, L.C.; SUZUKI, A. Efeitos do thidiazuron no tamanho e desenvolvimento dos frutos da macieira. Revista Brasileira de Fruticultura, Cruz das Almas, v.14, n.2, p.127-134, 1992.

REYNOLDS, A.G.; WARDLE, D.A.; ZUROWSKI, C.; LOONEY, N.E. Phenylureas CPPU and thidiazuron affect yield components, fruit composition, and storage potential of four seedless grape selections. Journal of the American Society for Horticultural Science, Alexandria, v.117, n.1, p.85-89, 1992.

RETAMALES, J.; BANGHERTH, F.; COOPER, T.; CALLEJAS, R. Effects of CPPU and $\mathrm{GA}_{3}$ on fruit quality of sultanina table grape. Acta Horticulturae, Leiden, n.394, 1995. 